\title{
Estudo Cinético da Reação Heterogênea de Despolimerização do PET Pós-Consumo em Meio Alcalino - Influência da Velocidade de Agitação*
}

\author{
Priscila S. Curti, Adhemar Ruvolo Fo \\ Departamento de Química, UFSCar
}

Resumo: Existe grande preocupação ambiental e econômica quanto à reutilização dos materiais poliméricos pós-consumo, devido ao grande volume de descarte destes nos lixos urbanos e ao valor agregado que esses materiais apresentam. Por isso existem vários estudos relacionados à pesquisa para o desenvolvimento de métodos de reciclagem mecânica e química dessa categoria de materiais, principalmente o PET. Neste trabalho o PET pós-consumo, obtido a partir de garrafas, foi submetido à reciclagem química usando solução de hidróxido de sódio em etilenoglicol. Foi verificado que o rendimento da reação e a cinética de despolimerização foram influenciadas pela velocidade de agitação do meio reacional. A $1360 \mathrm{rpm}$ a resistência ao processo de transferência de massa do produto sólido a partir da superfície das amostras de PET não reagidas para a solução foi eliminada e a reação passou a ser controlada pela etapa química. A equação cinética deduzida a partir do modelo considerando o meio heterogêneo apresentou boa concordância com os resultados experimentais e os valores das constantes de velocidade aparentes foram altos, comparados com valores de outros trabalhos da literatura. Estes resultados sugerem que o meio alcalino usado é bastante eficiente, pois a $1360 \mathrm{rpm}$ e a $170{ }^{\circ} \mathrm{C}$ apenas 6 min foram suficientes para obter $100 \%$ de tereftalato de dissódio.

Palavras-chave: PET pós-consumo, despolimerização, meio alcalino, cinética, meio heterogêneo.

\section{Kinetics Study of the Heterogeneous Depolymerization of Waste Pet in Alkaline Medium - Stirring Rate Effect}

\begin{abstract}
There is an environmental and economic concern related to recycling waste polymers, due to its high accumulation in the domiciliary urban waste and due to the aggregated value of these materials. Thus, many studies of mechanical and chemical recycling of waste plastics, mainly PET, have been proposed. In this work, waste PET obtained from bottles was submitted to chemical recycling using a solution of sodium hydroxide in ethylene glycol. The reaction extent and the kinetics were seen to be influenced by the stirring rate in the solution. At $1360 \mathrm{rpm}$, the mass transfer resistance of the product from the unreacted PET surface to the solution was eliminated, and the reaction rate became controlled by the chemical process. The kinetic equation derived from a model with a heterogeneous medium fitted the experimental results well, and the apparent rate constants were higher than those cited in the literature. These results suggest that the alkaline medium used is very efficient and only 6 minutes were sufficient to obtain $100 \%$ of sodium terephthalate yield at $1360 \mathrm{rpm}$ and $170{ }^{\circ} \mathrm{C}$.
\end{abstract}

Keywords: PET waste, depolymerization, alkaline medium, kinetics, heterogeneous medium.

\section{Introdução}

Entre os principais termoplásticos usados industrialmente, o Poli(tereftalato de etileno) (PET) apresenta a maior percentagem de descarte em volume nos lixos municipais brasileiros. No Brasil, a resina de PET virgem é empregada principalmente no setor de embalagens para bebidas carbonatadas, para o qual se destina atualmente em torno de $90 \%$ de sua produção ${ }^{[1]}$. Essas embalagens são rapidamente descartadas após o consumo e acumulam-se nos lixos urbanos. Por isso o processo de reciclagem torna-se uma alternativa muito importante tanto do ponto de vista econômico quanto ambiental. O PET é um polímero de condensação e pode ser submetido à reciclagem mecânica ou química ${ }^{[2]}$. A reciclagem química desse poliéster é bastante interessante do ponto de vista industrial. Os principais métodos de despolimerização usados são a hidrólise, a glicólise, a metanólise e a aminólise ${ }^{[3]}$. Quando a despolimerização do PET é realizada por meio da hidrólise, os produtos obtidos são o ácido tereftálico (TPA) e etileno glicol (EG). No Brasil, a resina de PET virgem é produzida principalmente a partir dessas substâncias ${ }^{[4]}$. O TPA também pode ser usado para a produção de resinas alquídicas, empregadas no setor de tintas. Assim a partir da reciclagem química do PET, a importação e o custo das matérias-primas, obtidas como produto da hidrólise, podem diminuir. No entanto as metodologias de

*Versão preliminar deste trabalho, na forma de resumo estendido, foi apresentada e premiada no $8^{\circ}$ Congresso Brasileiro de Polímeros, realizado em Águas de Lindóia, no período de 6 a 10 de novembro de 2005.

Autor para correspondência: Adhemar Ruvolo Filho, Grupo de Processamento e Propriedades em Polímeros, Centro de Ciências Exatas e de Tecnologia, Departamento de Química, UFSCar, Rodovia Washington Luiz, Km 235, Caixa Postal 676, CEP: 13565-905, São Carlos, SP, Brasil.

E-mail: adhemar@power.ufscar.br. 
hidrólise encontradas na literatura são dispendiosas. Para alcançar altos rendimentos da reação esses métodos necessitam do uso de soluções concentradas ${ }^{[5,6]}$, altas temperaturas ${ }^{[7-9]}$, catalisadores ${ }^{[8]}$ ou altas pressões ${ }^{[9,10]}$.

Em nosso grupo de pesquisa foi realizado um estudo da despolimerização do PET pós-consumo em meio alcalino ${ }^{[1]}$. Nesse estudo o PET foi convertido a TPA, com rendimento de $99 \%$, após 10 min de reação, a $170{ }^{\circ} \mathrm{C}$ e pressão atmosférica. Como o único solvente usado foi o etileno glicol, o mesmo pode ser facilmente recuperado pelo processo de destilação e retornar ao processo de despolimerização. Assim, esse método pode ser considerado bastante eficiente e satisfatório do ponto de vista industrial, comparado aos métodos anteriormente citados.

Abaixo da faixa do ponto de fusão do PET, que é entre 255 e $265^{\circ} \mathrm{C}$, a hidrólise é claramente uma reação heterogênea, ocorrendo na interface sólido-líquido. Por isso os efeitos na mudança da área superficial efetiva das amostras durante a despolimerização devem ser considerados no estudo cinético.

Vários autores realizaram o estudo cinético da reação de despolimerização do PET em temperaturas abaixo do ponto de fusão considerando o meio homogêneo ${ }^{[10,12-15]}$, o que pode levar a erros na dedução correta da equação cinética e conseqüentemente na interpretação dos parâmetros cinéticos.

Yoshioka e colaboradores aplicaram o modelo de diminuição da partícula sem reação no núcleo para realizar o estudo cinético da despolimerização do PET pós-consumo em pó em meio ácido usando solução de ácido nítrico $\left(\mathrm{HNO}_{3}\right)^{[5]}$ e posteriormente solução de ácido sulfúrico $\left(\mathrm{H}_{2} \mathrm{SO}_{4}{ }^{[6]}\right.$. Observaram um aumento da área superficial das amostras de PET não reagidas em função do aumento da conversão em TPA devido à formação de poros e rachaduras na superfície do PET. Adicionaram essa correção ao modelo original e verificaram que a equação cinética deduzida apresentou uma boa concordância com os dados experimentais. No entanto, obtiveram bons rendimentos somente após longos tempos de reação, em torno de $24 \mathrm{~h}$ a $100{ }^{\circ} \mathrm{C}$, usando solução de $\mathrm{HNO}_{3}$ 13 mol. $\mathrm{L}^{-1}$, e em torno de $1 \mathrm{~h}$ a $190{ }^{\circ} \mathrm{C}$ usando solução de $\mathrm{H}_{2} \mathrm{SO}_{4} 3$ mol.L $\mathrm{L}^{-1}$.

Kao e colaboradores ${ }^{[9]}$ estudaram a despolimerização da resina de PET virgem a temperaturas acima do seu ponto de fusão usando água como agente hidrolítico. Estudaram o efeito da velocidade de agitação do meio reacional na eficiência da reação de despolimerização. Realizaram experimentos entre as velocidades de agitação de 50 e $600 \mathrm{rpm}$, verificando que acima de $200 \mathrm{rpm}$ o processo de resistência à transferência de massa foi eliminado, de modo que a reação alcançou maiores conversões após $10 \mathrm{~min}$ de reação a $265^{\circ} \mathrm{C}$ e $400 \mathrm{rpm}$, mantendo a relação em massa de PET: água em 1:10. Esses tempos são menores comparados aos resultados de Yoshioka e col ${ }^{[5,6]}$. Nessas condições, os autores atribuíram o aumento na eficiência da despolimerização ao aumento da concentração de grupos carboxílicos durante a reação e à eli- minação do processo de resistência à transferência de massa do meio reacional em maiores velocidades de agitação.

No entanto, ainda não foi encontrado na literatura nenhum estudo sobre a influência do processo de transferência de massa no rendimento e cinética de despolimerização do PET abaixo do seu ponto de fusão. Nesse sentido, este trabalho tem como objetivo estudar a influência da velocidade de agitação do meio reacional na cinética da reação de hidrólise do PET pós-consumo em meio alcalino, em temperaturas abaixo do seu ponto de fusão, considerando o modelo cinético para sistemas heterogêneos.

\section{Experimental}

\section{Materiais}

O material utilizado foi o Poli(tereftalato de etileno) grau garrafa pós-consumo (PETr), cedido na forma de flakes pela indústria recicladora Embrapol. Esses flakes foram lavados, secos em estufa e peneirados para separação granulométrica. O material foi armazenado para o posterior uso em local fechado na temperatura de $23{ }^{\circ} \mathrm{C}$ e umidade relativa média do ar de 70\%. Também usou-se a resina de PET virgem S80 (PETV), cedida na forma de pellets pelo grupo Mossi \& Ghisolfi (Brasil). Para as reações de despolimerização também foram utilizados hidróxido de sódio $(\mathrm{NaOH})$ comercial e etileno glicol (EG) da Synth. Esses reagentes foram usados como recebido.

\section{Preparação das amostras de PETV e de PETr}

Os pellets de PETV foram previamente secos e cristalizados em estufa a $180{ }^{\circ} \mathrm{C}$ durante $4 \mathrm{~h}$ (PETVSEC) enquanto os flakes de PETr foram submetidos a três condições de pré-tratamento: i) secagem e cristalização em estufa a $180{ }^{\circ} \mathrm{C}$ durante quatro horas (PETrSEC); ii) exposição à umidade relativa do ar nas condições de armazenamento dos flakes (PETrREC); e iii) imersão em água até a saturação (PETrSAT). O teor de água contido nos pellets de PETVSEC e nos flakes de PETr submetidos aos três pré-tratamentos citados foi determinado por meio de análise termogravimétrica usando um equipamento TG 2050 da TA Instruments. As análises termogravimétricas foram realizadas em panela de platina, usando razão de aquecimento de $10^{\circ} \mathrm{C} \cdot \mathrm{min}^{-1}$ e fluxo de $\mathrm{N}_{2}$ de $10 \mathrm{~mL} \cdot \mathrm{min}^{-1}$ na balança e de $90 \mathrm{~mL} \cdot \mathrm{min}^{-1}$ no forno. A massa das distintas amostras usadas para essas análises foi de $19,16 \pm 0,16 \mathrm{mg}$. Os resultados dos teores de água obtidos por meio dessa análise são apresentados na Tabela 1.

Tabela 1. Teor de água contido nos pellets de PETVSEC e nos flakes de PETr, nas três condições de pré-tratamento citados.

\begin{tabular}{cc}
\hline Amostra & Teor de água (\%) \\
\hline pellets de PETVSEC & 0,00 \\
flakes de PETrREC & $0,21 \pm 0,02$ \\
flakes de PETrSEC & 0,00 \\
flakes de PETrSAT & $0,77 \pm 0,10$ \\
\hline
\end{tabular}


Os pellets de PETVSEC e os flakes de PETr, submetidos aos três diferentes pré-tratamentos, foram igualmente processados por compressão em prensa hidráulica Carver a $260{ }^{\circ} \mathrm{C}$ até a completa fusão do polímero e em seguida as placas moldadas foram submetidas a um resfriamento rápido a $0{ }^{\circ} \mathrm{C}$. As placas obtidas após o processamento foram denominadas PETVSECT, PETrSATT, PETrRECT e PETrSECT, respectivamente, as quais foram cortadas em amostras com formato geométrico de paralelepípedo, com as dimensões $0,8 \mathrm{~cm}$ de largura, $2,3 \mathrm{~cm}$ de comprimento e $0,27 \mathrm{~cm}$ de espessura.

\section{Reação de despolimerização}

As amostras de PETVSECT e de PETrT, obtidas após o processamento, foram submetidas à reação de despolimerização de acordo com a otimização realizada em trabalhos prévios desse grupo ${ }^{[11]}$ : usando solução de $\mathrm{NaOH}$ em etilenoglicol 1,1 mol.. ${ }^{-1}$ e mantendo a relação molar de NaOH:PET constante em 4:1 em todos os experimentos (considerando que $1 \mathrm{~mol}$ da unidade fundamental do PET é $192 \mathrm{~g}$ ). As reações de despolimerização foram realizadas em função do tempo de reação, a pressão atmosférica, mantendo a temperatura constante em $150{ }^{\circ} \mathrm{C}, 170^{\circ} \mathrm{C}$ e $185^{\circ} \mathrm{C}$, respectivamente. Foi usado um reator consistindo de um balão volumétrico de $100 \mathrm{~mL}$ imerso em banho de óleo de silicone, um condensador de refluxo e um termômetro. Após a solução reagente contida no balão volumétrico atingir a temperatura desejada, a amostra de PETVSECT ou de PETrT (obtidas após o processamento) foi adicionada à solução reagente e imediatamente passou-se a marcar o tempo de reação. Após atingir o tempo de reação desejado, a reação foi interrompida por imersão do balão de reação em banho de água e gelo. Após cada tempo de reação a massa da amostra de PET não reagida foi devidamente lavada com água, em seguida foi seca e sua converso $(\chi)$ foi determinada por gravimetria, segundo a Equação 1:

$$
\chi=\frac{m_{P E T_{i}}-m_{P E T_{i}}}{m_{P E T_{i}}}
$$

Onde $\mathrm{m}_{\mathrm{PET}_{\mathrm{i}}}$ corresponde à massa inicial da amostra de PET

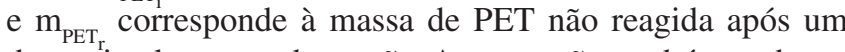
determinado tempo de reação. A conversão também pode ser apresentada em percentagem de conversão (\%).

\section{Caracterização do produto sólido obtido da reação de despolimerização}

O produto sólido obtido após a reação de despolimerização foi caracterizado por meio da técnica de análise elementar e pela técnica de espectrometria de massas com ionização por electrospray (IES-EM). Para a caracterização por análise elementar foi utilizado um equipamento Fisons EA 1108 CHNS, utilizando a técnica de cromatografia gasosa como ferramenta para a identificação dos elementos químicos. A análise por espectrometria de massas foi realizada em um equipamento UltrOTOF-Q (Brucker, Daltonics Billerica MA, USA). A amostra foi introduzida em uma bomba injetora com fluxo de $10 \mu \mathrm{L} \cdot \mathrm{min}^{-1}$. Para a investigação sistemática no modo negativo ${ }^{[16]} \mathrm{o}$ capilar foi mantido na temperatura de $250{ }^{\circ} \mathrm{C}$ e o potencial aplicado foi de $3 \mathrm{kV}$.

\section{Influência da velocidade de agitação do meio reacional na reação de despolimerização}

A influência da velocidade de agitação do meio reacional foi avaliada tanto na determinação do tempo para atingir o máximo de conversão quanto na estimativa da variação da área superficial efetiva em função da conversão das amostras de PETrSATT. A área superficial efetiva das amostras de PETrSATT, antes e após cada tempo de despolimerização, foi determinada por meio da isoterma BET usando um equipamento ASAP 2000, da Micromeritics. O gás usado como adsorvente foi o nitrogênio. Para as análises de área superficial efetiva $\left(\mathrm{S}_{\mathrm{ef}}\right)$ as amostras de PETrSATT reagidas em diferentes tempos de reação, conforme comentado anteriormente, foram lavadas com água em abundância, secas e mantidas em estufa a vácuo a $30^{\circ} \mathrm{C}$ até o momento da análise, de modo a eliminar toda a umidade da superfície que poderia interferir nas medidas. Todas as medidas de $\mathrm{S}_{\text {ef }}$ foram normalizadas em relação ao respectivo valor da área superficial efetiva inicial $\left(\mathrm{S}_{\mathrm{o}}\right)$.

A análise morfológica das superfícies das amostras de PETrSATT submetidas à reação de despolimerização a $170{ }^{\circ} \mathrm{C}$ em diferentes velocidades de agitação do meio reacional foi realizada utilizando um microscópio eletrônico Zeiss modelo DSM $940 \mathrm{~A}$. As amostras de PETrSATT não foram lavadas após cada tempo de reação, a fim de observar a ocorrência ou não de saturação da superfície polimérica não reagida com o produto da reação. Essas amostras foram recobertas com uma fina camada de ouro. A corrente e a potência do feixe foram de $80 \mu \mathrm{A}$ e de $20 \mathrm{kV}$, respectivamente. A análise morfológica foi realizada na temperatura de $22{ }^{\circ} \mathrm{C}$.

Os valores da razão $\mathrm{S}_{\mathrm{ef}} / \mathrm{S}_{\mathrm{o}}$ estimadas para as amostras de PETrSATT foram usadas no tratamento cinético da reação de despolimerização considerando o meio heterogêneo. As imagens de MEV de superfície das amostras de PETrSATT foram usadas para verificar a consistência dos resultados experimentais ao modelo cinético adotado.

\section{Resultados e Discussão}

Como citado na seção Experimental, as amostras de PETVSECT, PETrRECT, PETrSECT e de PETrSATT foram submetidas à hidrólise alcalina, usando solução de $\mathrm{NaOH}$ em EG anidro. Segundo a literatura ${ }^{[17]}$ a hidrólise alcalina de um éster é uma reação irreversível. O íon hidróxido ataca o carbono do grupo carbonila do éster, formando álcool e o sal do seu respectivo ácido. Assim a hidrólise alcalina do PET deveria formar o sal do ácido tereftálico, tereftalato de dissódio ( $\mathrm{Na}_{2}$-TPA) e o etileno glicol (EG), como produtos da reação. Após a despolimerização, o produto da reação foi acidificado e posteriormente neutralizado. As percentagens de carbono e hidrogênio do produto sólido foram determinadas por meio de análise elementar. Os resultados obtidos são apresentados na Tabela 2, juntamente com os valores teóricos das percen- 
Tabela 2. Valores teóricos e experimentais das percentagens dos elementos químicos carbono, hidrogênio e oxigênio, obtidos para o ácido tereftálico (TPA), por meio de análise elementar.

\begin{tabular}{lcc}
\hline Elemento & Teórica (\%) & $\begin{array}{c}\text { Experimental } \\
(\boldsymbol{\%})\end{array}$ \\
\hline Carbono & 57,83 & $59,40 \pm 0,10$ \\
Hidrogênio & 3,62 & $3,67 \pm 0,12$ \\
Oxigênio & 38,55 & $36,92 \pm 0,03$ \\
\hline
\end{tabular}

tagens de carbono e hidrogênio para o TPA. Pode ser observado que o valor experimental da percentagem de hidrogênio é semelhante ao valor teórico. No entanto observa-se que o valor da percentagem experimental de carbono é $2,7 \%$ maior em relação ao valor teórico, correspondendo a uma pureza de $97,3 \%$. Essa variação na percentagem de carbono provavelmente é devido à presença de carbonatos no produto da reação, provenientes da solução alcalina utilizada para a despolimerização, que foi preparada a partir do hidróxido de sódio comercial. A partir da análise de espectrometria de massas do produto sólido, foi obtido o valor de $\mathrm{m} / \mathrm{z}$ de 165,0199 para o pico do íon molecular (valor teórico de $\mathrm{m} / \mathrm{z}=165,0188$ ), correspondendo à desprotonação de um grupo carboxílico do ácido tereftálico $(\mathrm{m} / \mathrm{z}=166)$, segundo a reação apresentada na Figura 1. O erro obtido dessa análise foi de apenas $6 \mathrm{ppm}$, indicando que o produto da reação de despolimerização é, de fato, o ácido tereftálico (TPA). Por isso os produtos obtidos da reação de despolimerização do PET foram unicamente $\mathrm{Na}_{2}$-TPA (após a acidificação, TPA) e o EG.

Considerando as diferentes condições de pré-tratamento que os flakes de PETr foram submetidos antes do processamento, os quais possuíam diferentes teores de água, conforme apresentado na seção Experimental, pôde-se avaliar o efeito do teor de água na reatividade das amostras processadas por termoformagem frente ao meio alcalino durante a reação de despolimerização, comparado às amostras de PETVSECT. Os resultados dessa análise são apresentados na Figura 2. Verifica-se que a conversão das amostras de PETrSATT em tereftalato de dissódio ( $\mathrm{Na}_{2}$-TPA) ocorre em um intervalo de 6 min. O tempo máximo de conversão das amostras de PETrRECT é de $10 \mathrm{~min}$, e das amostras de PETVSECT e de PETrSECT é em torno de $22 \mathrm{~min}$. Esse resultado indica que a água interfere na reatividade das amostras processadas, sendo que quanto maior o teor de água nos flakes originais, maior é a reatividade observada nas amostras após o processamento por termoformagem.

Segundo a literatura a água atua como agente de degradação hidrolítica do PET a temperaturas acima do seu ponto de fusão, formando grupos químicos mais polares, tais como o ácido carboxílico ${ }^{[7,18]}$ e o acetaldeído ${ }^{[19,20]}$. Dessa forma, os flakes de PETr com maior teor de água absorvida possuem maior tendência à formação desses grupos químicos mais polares durante o processamento por termoformagem, principalmente em sua superfície, aumentando a sua reatividade frente ao íon hidroxila durante a despolimerização. Essa simples etapa antes da reação melhora a eficiência da despo-

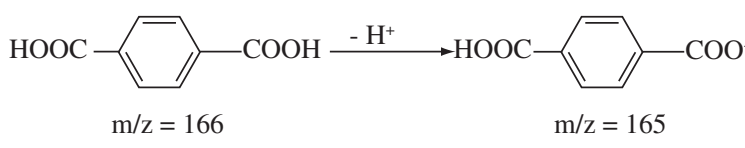

Figura 1. Reação de desprotonação do ácido tereftálico (TPA).

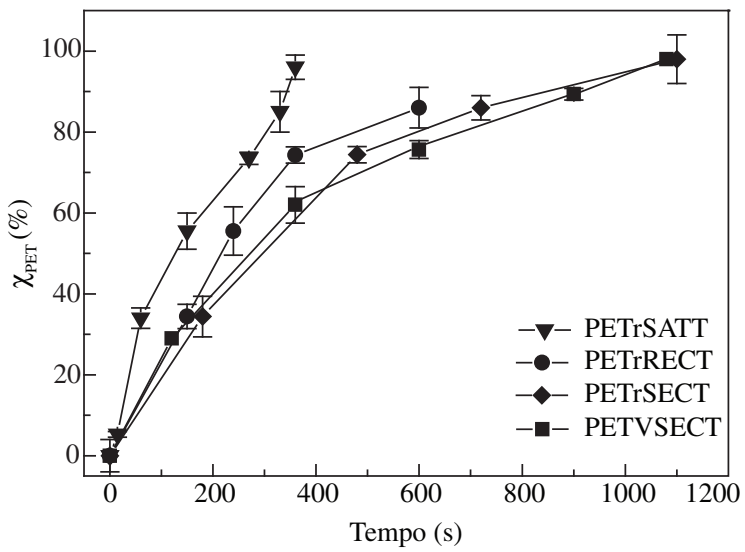

Figura 2. Efeito do teor de água presente nos pellets de PETVSEC e nos flakes de PETr, antes do processamento, no tempo para atingir o máximo de conversão a $170{ }^{\circ} \mathrm{C}$ e $1360 \mathrm{rpm}$ para as amostras processadas de PETVSECT, PETrSATT, PETrRECT e PETrSECT.

limerização, reduzindo acentuadamente o tempo necessário para alcançar a conversão máxima. Após essa constatação, somente amostras de PETrSATT foram usadas para as análises posteriores.

Em sistemas heterogêneos a velocidade de agitação do meio reacional interfere nos parâmetros cinéticos, devido a mudanças no tipo de estágio controlador da reação $0^{[21]}$. A velocidades de agitação muito baixas, o produto formado na superfície do sólido reagente pode não ser extraído de forma satisfatória, permanecendo aderido à superfície das amostras não reagidas. O produto sólido depositado sobre a superfície da amostra não reagida atuará como uma resistência dificultando a difusão dos íons hidroxila do meio líquido até a superfície da amostra não reagida, o que provavelmente reduz a velocidade da reação de despolimerização. Nessas condições o processo de transferência de massa interfere na reação e a mesma passa a ser controlada pelo fenômeno de difusão do produto sólido formado na superfície da amostra não reagida para a solução. Quando a velocidade de agitação atinge um valor tal que todo o produto formado na superfície da amostra não reagida é efetivamente extraído para a solução, o processo de transferência de massa não interfere na reação e a cinética da mesma passa a ser controlada pela etapa química.

$\mathrm{Na}$ Figura 3 são apresentados os resultados de conversão em função da velocidade de agitação do meio reacional para as reações de despolimerização das amostras de PETrSATT realizadas a $170{ }^{\circ} \mathrm{C}$. Nessa Figura é observado que a velocidade de agitação do meio reacional influencia o tempo para a reação atingir o máximo de conversão. Em baixas velocidades, como a $110 \mathrm{rpm}$, o tempo para atingir a conversão máxima é em torno de 16 min, enquanto o tempo para atingir a mesma conversão a $1360 \mathrm{rpm}$ é de apenas $6 \mathrm{~min}$. 


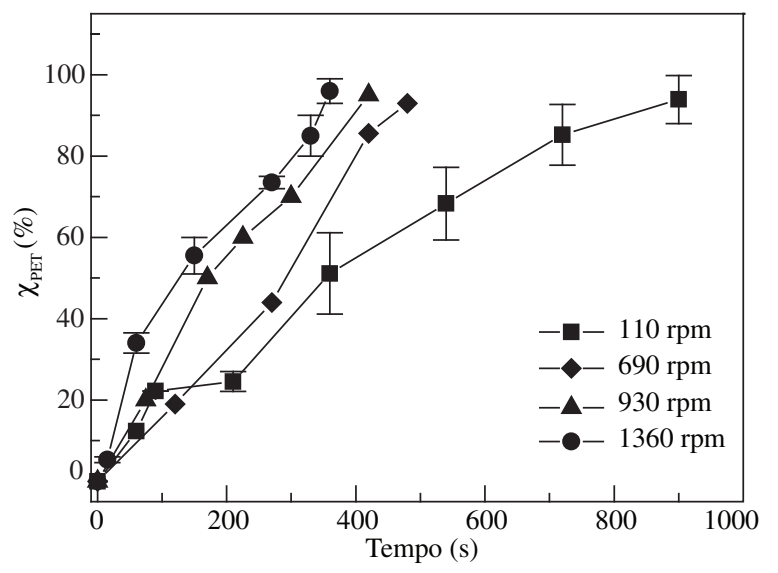

Figura 3. Efeito da velocidade de agitação do meio reacional no tempo para atingir o máximo de conversão para as amostras de PETrSATT submetidas à despolimerização a $170{ }^{\circ} \mathrm{C}$.

Essa variação no tempo necessário para atingir o máximo de conversão em função da mudança na velocidade de agitação está relacionada ao estágio controlador da reação de despolimerização. A $110 \mathrm{rpm}$ provavelmente o processo que controla a reação é a difusão do produto da superfície da amostra de PET não reagida para a solução. A 1360 rpm, provavelmente o cisalhamento é tal que número de Reynolds torna-se suficientemente alto para favorecer o processo de saída do $\mathrm{Na}_{2}$-TPA da superfície das amostras de PET não reagidas para a solução. Nessa situação a reação passa a ser controlada pela etapa química.

As micrografias eletrônicas de varredura de superfície das amostras de PETrSATT antes de sua submissão à reação de despolimerização e após sua despolimerização até uma conversão de $70 \%$ a $170{ }^{\circ} \mathrm{C}$, nas velocidades de agitação de 110 e 1360 rpm, são apresentadas na Figura 4. Observa-se que a superfície do PETrSATT obtida do processamento (Figura 4a) apresenta defeitos homogeneamente distribuídos. Após o tempo de reação necessário para atingir uma conversão de $70 \%$ ocorrem mudanças nas morfologias das superfícies das amostras de PETrSATT despolimerizadas em cada velocidade de agitação. A imagem de MEV da amostra de PETrSATT reagida a $110 \mathrm{rpm}$ (Figura 4b) apresenta a superfície totalmente recoberta pelo $\mathrm{Na}_{2}$-TPA enquanto a imagem da superfície da amostra de PETrSATT submetida à reação a 1360 rpm (Figura 4c) não apresenta vestígios do $\mathrm{Na}_{2}$-TPA aderido em sua superfície.

A razão da área superficial efetiva normalizada $\left(\mathrm{S}_{\mathrm{ef}} / \mathrm{S}_{\mathrm{o}}\right)$ em função da conversão das amostras de PETrSATT, submetidas à despolimerização a $170{ }^{\circ} \mathrm{C}$ em três diferentes velocidades de agitação, estão apresentados na Figura 5. Até aproximadamente $40 \%$ de conversão as curvas são semelhantes. Acima desse valor, a variação da área superficial efetiva normalizada passa a depender da velocidade de agitação do meio reacional, refletindo a dependência do estágio controlador da reação em relação à velocidade de agitação do meio reacional. A $110 \mathrm{rpm}$ a razão $\mathrm{S}_{\mathrm{ef}} / \mathrm{S}_{\mathrm{o}}$ é linear e relativamente baixa, devido à saturação da superfície da amostra
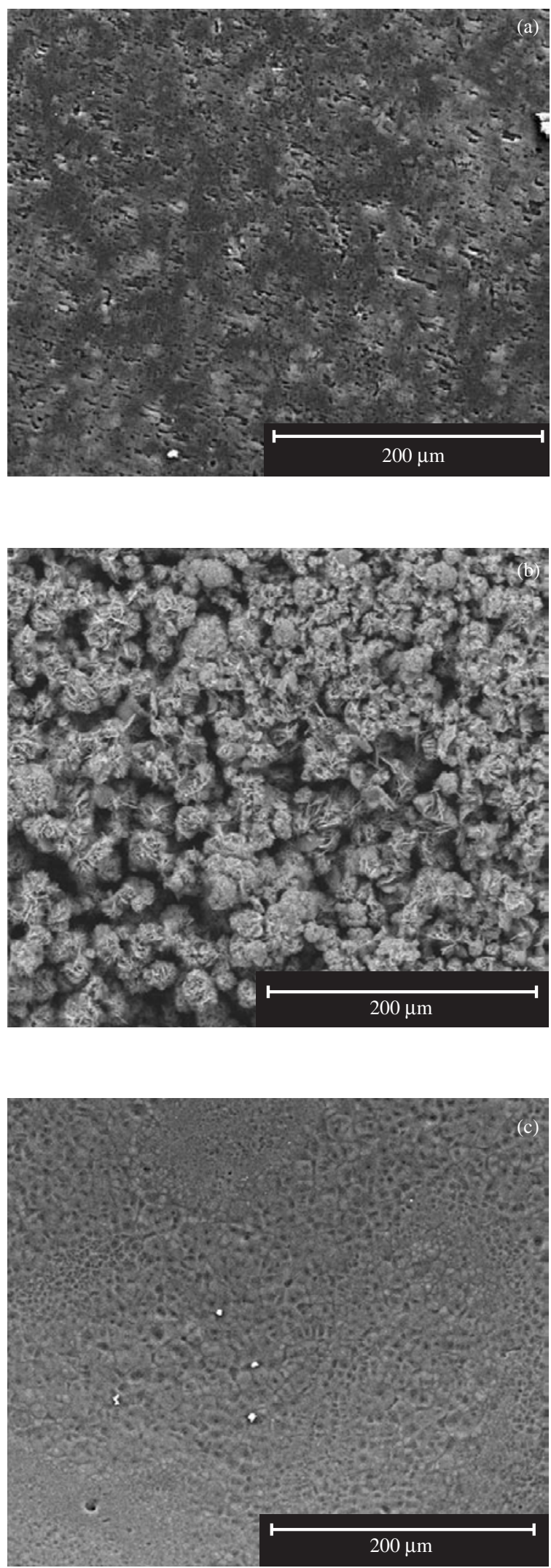

Figura 4. Micrografias eletrônicas de varredura de superfície das amostras de PETrSATT (a) antes da reação de despolimerização e após serem submetidas à despolimerização a $170{ }^{\circ} \mathrm{C}$ até atingirem $70 \%$ de conversão nas velocidades de agitação de: (b) $110 \mathrm{rpm}$ (9 min) e (c) $1360 \mathrm{rpm}$ (4 min). Todas as micrografias possuem ampliação de 200x. 


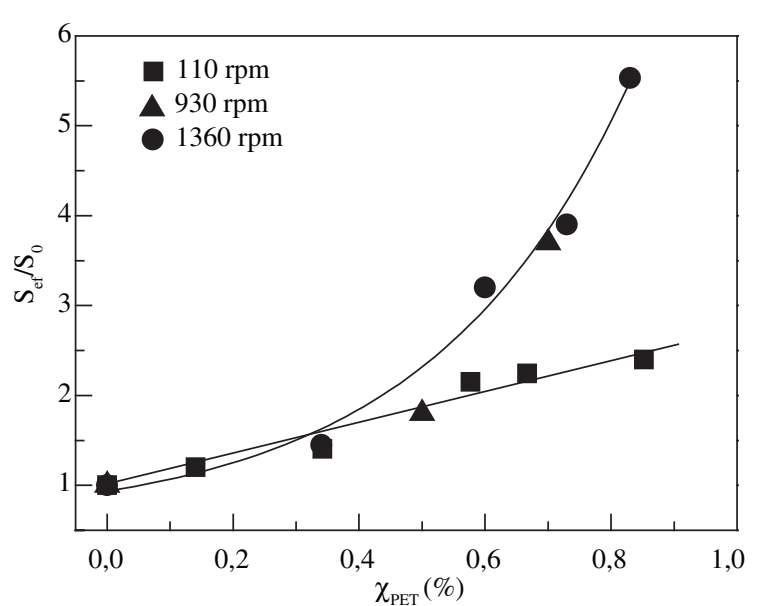

Figura 5. Razão $\mathrm{S}_{\mathrm{e} / \mathrm{S}} / \mathrm{S}_{\mathrm{o}}$ em função da conversão $(\chi)$ das amostras de PETrSATT submetidas à reação de despolimerização a $170{ }^{\circ} \mathrm{C}$ em diferentes velocidades de agitação do meio reacional.

não reagida com o produto formado durante a despolimerização. Quando a velocidade de agitação é aumentada para 930 e $1360 \mathrm{rpm}$, a razão $\mathrm{S}_{\mathrm{ef}} / \mathrm{S}_{\mathrm{o}}$ passa a ser exponencial, indicando que nessa situação o produto da reação é extraído da superfície da amostra não reagida concomitantemente à reação, tornando-a mais reativa e mais suceptível a constantes ataques dos grupos hidroxila presentes na solução reagente. Esse resultado mais uma vez reflete a influência da velocidade de agitação do meio reacional no estágio controlador da reação, corroborando com os resultados das Figuras 3 e 4.

Após essas análises, pôde-se garantir que as reações de despolimerização realizadas na velocidade de agitação de $1360 \mathrm{rpm}$ foram controladas pela etapa química, de modo que o processo de resistência à transferência de massa do produto a partir da superfície da amostra de PETrSATT não reagida para a solução passou a não interferir na reação.

\section{Estudo cinético da reação de despolimerização das amostras de PETrSATT}

Na Figura 6 são apresentadas as micrografias eletrônicas de varredura de superfície das amostras de PETrSATT submetidas à despolimerização a $170{ }^{\circ} \mathrm{C}$ e $1360 \mathrm{rpm}$ em função do tempo de reação.

Na Figura 6a verifica-se que a superfície da amostra de PETrSATT apresenta defeitos distribuídos. Após 1 min de reação, a amostra alcança 30\% de conversão e a micrografia correspondente à sua superfície (Figura 6b) indica um aumento de poros e rachaduras, os quais tornam-se maiores quando a conversão alcança 50\% (Figura 6c). No entanto após 70\% de conversão, a micrografia da superfície do PETrSATT não reagido (Figura 6d) aparentemente não apresenta poros evidentes, devido ao processo de despolimerização ocorrer por eliminação de camadas da superfície polimérica. No entanto a ampliação dessa imagem (Figura 6e) mostra claramente o aumento do número de poros com diâmetros menores na superfície da amostra de PETrSATT, e conseqüentemente essa maior quantidade de poros aumenta a reatividade da mesma nos instantes finais da reação. Por isso, para realizar o estudo cinético dessa reação em meio heterogêneo foi adotado o modelo de conversão da partícula sem reação no núcleo. Yoshioka e colaboradores ${ }^{[5,6]}$ propuseram uma correção a esse modelo, pois observaram um aumento linear entre a área superficial efetiva das amostras de PET não reagidas e a sua conversão. Esse aumento ocorreu devido à formação de poros e rachaduras na superfície das amostras de PET não reagidas. Aplicaram o modelo corrigido a amostras com geometria esférica. Nesse trabalho, o modelo cinético proposto é aplicado a amostras com formato de paralelepípedo, devido ser a forma geométrica mais próxima das amostras obtidas no processo de moagem industrial das garrafas de bebidas carbonatadas recicladas.

Considerando que as amostras de PETrSATT possuem o volume de um paralelepípedo e aplicando o modelo cinético correspondente a reação na superfície de um sólido em um meio líquido, chega-se à Equação 2:

$$
\frac{\mathrm{d}\left(\mathrm{x}_{\mathrm{o}} \mathrm{y}_{\mathrm{z}} \mathrm{z}_{t} \rho\right)}{\mathrm{dt}}=-k C_{A} S_{e f}
$$

Onde o termo $\mathrm{x}_{\mathrm{o}} \mathrm{y}_{\mathrm{o}} \mathrm{z}_{\mathrm{t}}$ corresponde ao volume de um paralelepípedo em um instante, $t$, qualquer da reação. $\mathrm{O}$ volume inicial da amostra é dado por $\mathrm{x}_{\mathrm{o}} \mathrm{y}_{\mathrm{o}} \mathrm{z}_{\mathrm{o}}$, onde $\mathrm{x}_{\mathrm{o}}$ é o comprimento inicial; $\mathrm{y}_{\mathrm{o}}$ é a largura inicial e $\mathrm{z}_{\mathrm{o}}$ é a espessura inicial; sendo ainda $\rho \mathrm{a}$ densidade da amostra de PETrSATT, $\mathrm{k}$ a constante de velocidade de diminuição da amostra, $\mathrm{C}_{\mathrm{a}}$ a concentração de hidróxido de sódio e $\mathrm{S}_{\text {ef }}$ a área superficial efetiva da amostra sólida disponível para sofrer a reação de despolimerização.

A variação na espessura $z_{t}$ pode ser considerada como a variável cinética relacionada à conversão, pela expressão:

$$
\chi=1-\frac{x_{o} y_{o} z_{t} \rho}{x_{o} y_{o} z_{o} \rho}
$$

A área superficial efetiva é dada pela expressão:

$$
\mathrm{S}_{\mathrm{ef}}=\mathrm{S}_{\mathrm{geom}}(1-\chi)(\text { termo de correção) }
$$

A área geométrica inicial $\left(\mathrm{S}_{\text {geom }}\right)$ é dada pela área total do paralelepípedo, a qual é:

$$
S_{\text {geom }}=2\left(x_{o} z_{o}+y_{o} z_{o}+x_{o} y_{o}\right)
$$

Como mostrado na expressão 4, a área superficial efetiva é multiplicada pela área geométrica inicial da amostra, pela fração da amostra de PET não reagida após determinados tempos de reação e também é multiplicada por um termo de correção relacionado à variação real da área superficial efetiva em função da conversão das amostras. Por isso é necessário avaliar como essa área superficial efetiva varia em função da conversão das amostras de PETrSATT. Como comentado anteriormente, Yoshioka e colaboradores ${ }^{[6]}$ encontraram uma relação crescente e linear para a variação da área superficial efetiva das amostras de PET em função de sua conversão. Por isso adicionaram um termo de correção linear à equação de velocidade. Na Figura 7 são apresentados os resultados da 

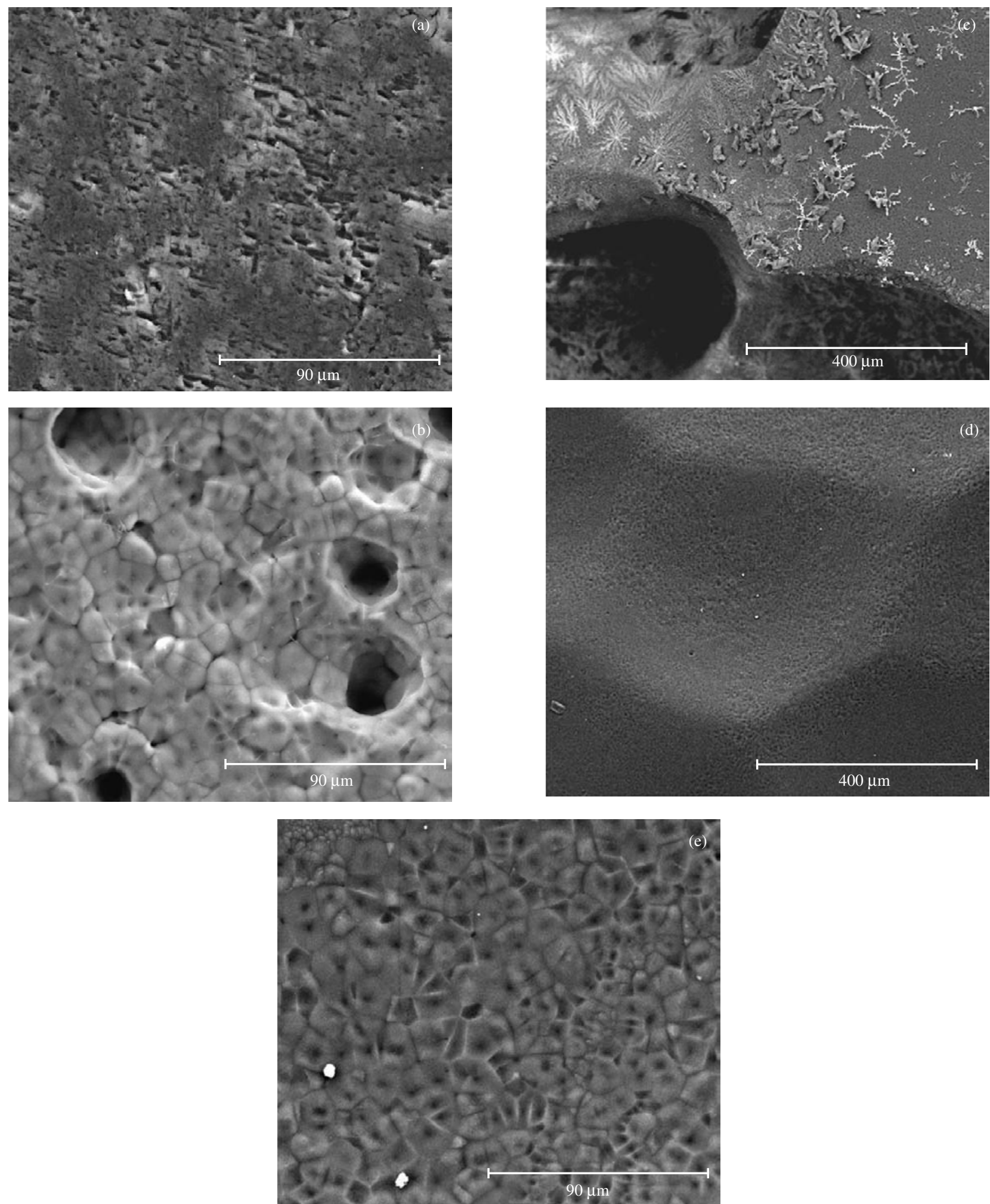

Figura 6. Micrografias eletrônicas de varredura de superfície das amostras de PETrSATT: a) antes da reação de despolimerização (500x) e após serem submetidas à reação de despolimerização a $170{ }^{\circ} \mathrm{C}$ e $1360 \mathrm{rpm}$ durante: b) 1 min (500x); c) 2 min e meio (100x); d) 4 min (100x); e e) 4 min (500x).

razão $\mathrm{S}_{\mathrm{et}} / \mathrm{S}_{\mathrm{o}}$ em função da conversão das amostras de $\mathrm{PETr}$ SATT submetidas à despolimerização. De acordo com essa Figura, a razão $\mathrm{S}_{\mathrm{ef}} / \mathrm{S}_{\mathrm{o}}$ em função da conversão é exponencial, indicando a formação e o aumento do número de defeitos na superfície das amostras de PETrSATT não reagidas. Esse aumento de defeitos na superfície da amostra acelera a conversão, provavelmente devido ao aumento de grupos polares reativos durante a despolimerização. 


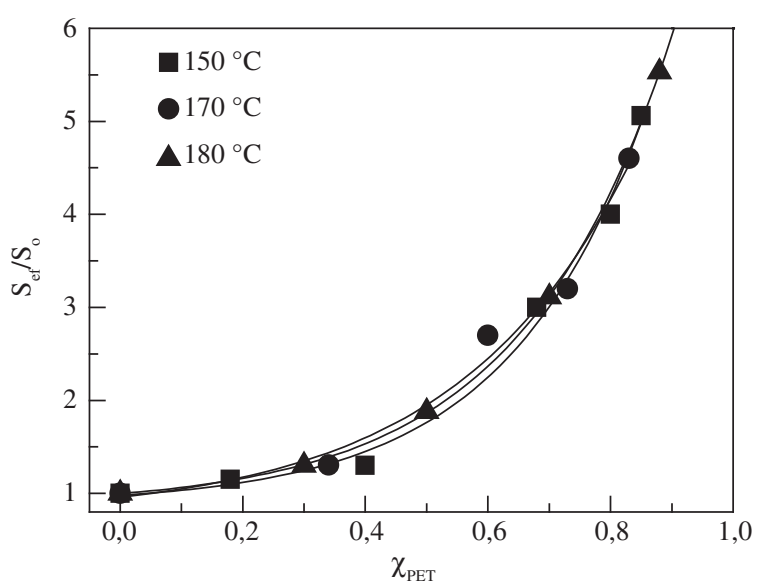

Figura 7. Razão $S_{e f} / S_{o}$ em função da conversão $(\chi)$ das amostras de PETrSATT submetidas à reação de despolimerização a 1360 rpm nas temperaturas de 150,170 e $185^{\circ} \mathrm{C}$.

Alguns autores ${ }^{[10,12-15]}$ também realizaram estudos cinéticos da despolimerização do PET em temperaturas abaixo de seu ponto de fusão e trataram o sistema heterogêneo como homogêneo considerando a área superficial das amostras constante durante toda a reação. No entanto a partir dos estudos de Yoshioka ${ }^{[5,6]}$ e dos resultados apresentados até o momento nesse trabalho, fica evidente a importância de avaliar a relação entre a variação da área superficial efetiva e a conversão das amostras de PET.

A partir da Figura 7 verifica-se outra informação importante, qual seja, independente da temperatura da reação, no intervalo entre 150 e $185^{\circ} \mathrm{C}$, a razão $\mathrm{S}_{\text {ef }} / \mathrm{S}_{\mathrm{o}}$ possui o mesmo comportamento exponencial. O fato da razão $\mathrm{S}_{\mathrm{er}} / \mathrm{S}_{\mathrm{o}}$ ser independente da temperatura indica que, mantendo as dimensões das amostras constantes, a temperatura afeta apenas o tempo necessário para atingir o máximo de conversão, mas não afeta as características de reatividade superficial das amostras. Assim, pode-se obter a curva $\mathrm{S}_{\mathrm{ef}} / \mathrm{S}_{\mathrm{o}}$ em função de $\chi$, em uma temperatura e estender a outras temperaturas, dentro do intervalo estudado, sem a necessidade de realizar novos experimentos. Essa característica torna esse método muito interessante do ponto de vista industrial.

O termo de correção exponencial foi obtido a partir do melhor ajuste dos pontos das curvas apresentadas na Figura 6, sendo igual a aexp $(\chi / c)$, onde $\chi$ é a conversão de PETrSATT ; a e c são constantes, cujos valores numéricos são 0,09 e 0,23, respectivamente. Assim, para amostras de PET na forma geométrica de paralelepípedo, a Equação 4 torna-se:

$$
S_{e f}=2\left(x_{o} z_{o}+y_{o} z_{o}+x_{o} y_{o}\right) a \exp ^{\chi / c}(1-\chi)
$$

Substituindo a Equação 6 na Equação 2, com a finalidade de corrigir a variação verdadeira da área superficial das amostras de PETrSATT em função da conversão, obtém-se:

$$
-\frac{d\left(x_{o} y_{o} z_{t} \rho\right)}{d t}=
$$

$$
2 a k C_{A}\left(x_{o} z_{o}+y_{o} z_{o}+x_{o} y_{o}\right)(1-\chi) \exp ^{x / c}
$$

Mas, da Equação 3 tem-se que:

$$
(1-\chi)=\frac{x_{o} y_{o} z_{t} \rho}{x_{o} y_{o} z_{o} \rho}
$$

Tomando a derivada de (8):

$$
\frac{d(1-\chi)}{d t}=\frac{1}{x_{o} y_{o} z_{o} \rho} \frac{d\left(x_{o} y_{o} z_{t} \rho\right)}{d t}
$$

Substituindo (7) em (9):

$$
\begin{aligned}
& -\frac{d(1-\chi)}{d t}= \\
& \frac{2 a k C_{A}}{x_{o} y_{o} z_{o} \rho}\left(x_{o} z_{o}+y_{o} z_{o}+x_{o} y_{o}\right)(1-\chi) \exp ^{\chi / c}
\end{aligned}
$$

Integrando a Equação 11 ${ }^{[22]}$, obtém-se:

$$
-\frac{(1-\chi)}{c}-\ln (1-\chi)+\frac{1}{c}=k_{v} t
$$

Onde $\mathrm{k}_{\mathrm{v}}$ é a constante de velocidade aparente da reação, a qual é dada por:

$$
k_{v}=\frac{2 a k C_{A} \exp ^{1 / \mathrm{c}}}{x_{o} y_{o} z_{o} \rho}\left(x_{o} z_{o}+y_{o} z_{o}+x_{o} y_{o}\right)
$$

A Equação 11 é a equação cinética integrada deduzida a partir da consideração das amostras de PETrSATT com formato geométrico de paralelepípedo. Essa equação foi aplicada aos dados experimentais e os resultados cinéticos são apresentados na Figura 8. Foram obtidas correlações lineares nas três temperaturas estudadas, indicando que o modelo cinético adotado possui boa concordância com os dados experimentais.

A partir da regressão linear dos dados cinéticos da Figura 8, foram calculadas as constantes de velocidade aparente, $\mathrm{k}_{\mathrm{v}}$, da reação de hidrólise do PETrSATT em meio alcalino. Esses resultados são apresentados na Tabela 3. A partir de uma representação de Arrhenius, a energia de ativação aparente, $\mathrm{E}_{\mathrm{a}}$, foi calculada em $172,71 \mathrm{~kJ} \cdot \mathrm{mol}^{-1}$. Esse valor é alto, comparado aos valores de energia de ativação de outros estudos de despolimerização do PET encontrados na literatu$\mathrm{ra}^{[5,6,9,10,12-15]}$, os quais usaram metodologias diferentes para o tratamento cinético.

Considerando que alguns autores trataram o meio heterogêneo como homogêneo ${ }^{[10,12-15]}$, provavelmente os valores das constantes de velocidade aparente e da energia de ativação não sejam totalmente realísticos. Yoshioka e colaboradores realizaram a despolimerização das amostras de PET em meio ácido considerando o meio como heterogêneo ${ }^{[5,6]}$. Esses autores avaliaram o efeito da concentração de íons $\mathrm{H}_{3} \mathrm{O}^{+}$na cinética de despolimerização. Para isso relacionaram o logaritmo das constantes de velocidade aparente, $\mathrm{k}_{\mathrm{v}}$, e os valores da função de Hammett calculados para as diferentes concentrações da solução de $\mathrm{H}_{2} \mathrm{SO}_{4}$, que variou entre 3 mol. $\mathrm{L}^{-1}$ e 9 mol. $\mathrm{L}^{-1}$. 


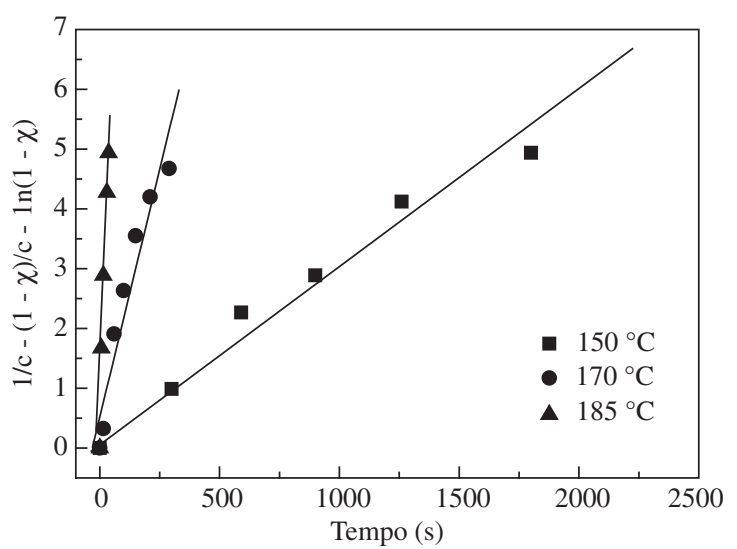

Figura 8. Cinética da reação de despolimerização das amostras de PETrSATT realizadas a $1360 \mathrm{rpm}$ nas temperaturas de 150,170 e $185^{\circ} \mathrm{C}$.

Tabela 3. Valores de constante de velocidade aparente, $\mathrm{k}_{\mathrm{v}}$ e energia de ativação, $\mathrm{E}$, obtidos a partir da equação cinética 11.

\begin{tabular}{ccc}
\hline Temperatura $\left({ }^{\circ} \mathbf{C}\right)$ & $\mathbf{k}_{\mathbf{v}}\left(\mathbf{h}^{-1}\right)$ & $\mathbf{E}_{\mathbf{a}}\left(\mathbf{k J ~ m o l}{ }^{-1}\right)$ \\
\hline 150 & 10,08 & \\
170 & 59,65 & 172,7 \\
185 & 453,46 & \\
\hline
\end{tabular}

Observaram uma dependência direta e linear entre a função de Hammett e os valores de $\mathrm{k}_{\mathrm{v}}$. Isto sugere que a reação na superfície sólida do PET, catalisada por íons $\mathrm{H}_{3} \mathrm{O}^{+}$, é a etapa determinante da reação e a constante de velocidade aparente é diretamente proporcional à concentração de íons $\mathrm{H}_{3} \mathrm{O}^{+}$presentes no meio reacional. $\mathrm{O}$ valor de energia de ativação encontrada por esses autores foi menor, comparada ao valor de energia de ativação determinado nesse respectivo trabalho, no qual as reações foram realizadas em meio alcalino. No entanto esses autores não avaliaram o efeito da agitação do meio reacional na cinética da reação. Os tempos necessários para atingir a conversão máxima observados por Yoshioka e colaboradores são muito mais longos, comparados aos resultados obtidos neste trabalho. Pode ser que nas condições de agitação estudadas, a resistência à transferência de massa não tenha sido satisfatoriamente eliminada e provavelmente a reação ocorreu em maiores tempos devido a alguma saturação da superfície com o produto. Por isso torna-se mais importante comparar os valores de $\mathrm{k}_{\mathrm{v}}$ desses processos.

Yoshioka e colaboradore ${ }^{[6]}$ avaliaram o efeito do diâmetro das amostras de PET e da concentração de $\mathrm{H}_{2} \mathrm{SO}_{4}$ na cinética da reação de despolimerização do PET. Primeiro mantiveram a concentração da solução constante em 5 mol. $\mathrm{L}^{-1}$ e comparam o efeito do diâmetro inicial das amostras de PET nos parâmetros cinéticos da reação. De acordo com os resultados desses autores, os valores de $\mathrm{k}_{\mathrm{v}}$ obtidos para a reação de despolimerização realizada a $150{ }^{\circ} \mathrm{C}$, usando solução de $\mathrm{H}_{2} \mathrm{SO}_{4} 5$ mol. $\mathrm{L}^{-1}$ foram em torno de $0,196 \mathrm{~h}^{-1}$ e $0,168 \mathrm{~h}^{-1}$, usando amostras de PET com diâmetro de 0,09 e $0,135 \mathrm{~mm}$, respectivamente. Verifica-se que o valor da concentração da solução de $\mathrm{H}_{2} \mathrm{SO}_{4}$ é cinco vezes maior e a dimensão das amostras de PET é de vinte a trinta vezes maior, em relação ao valor da concentração da solução de $\mathrm{NaOH}$ e da dimensão de espessura das amostras de PET usadas neste trabalho, enquanto o valor de $\mathrm{k}_{\mathrm{v}}$ obtido nesse trabalho é cerca de cinquienta a sessenta vezes maior, comparados aos valores de $\mathrm{k}_{\mathrm{v}}$ determinados por Yoshioka e colaboradores nessas condições indicadas. Comparando-se ainda o valor de $\mathrm{k}_{\mathrm{v}} \mathrm{de}$ $0,058 \mathrm{~h}^{-1}$ obtido por esses autores para a reação de despolimerização do PET realizada na temperatura de $170{ }^{\circ} \mathrm{C}$ usando solução de $\mathrm{H}_{2} \mathrm{SO}_{4} 3$ mol. $\mathrm{L}^{-1}$, verifica-se que para uma concentração de $\mathrm{NaOH}$ três vezes menor, o valor de $\mathrm{k}_{\mathrm{v}}$ determinado neste trabalho, na mesma temperatura, é mil vezes maior.

Assim a partir dessas comparações pode-se verificar que o meio reacional alcalino, sob condições de agitação adequadas, é mais eficiente e comercialmente mais vantajoso comparado ao meio ácido citado por Yoshioka e colaboradores.

\section{Conclusões}

A partir dos experimentos realizados, conclui-se que o estudo da influência da velocidade de agitação do meio reacional é importante para o meio heterogêneo em questão. Verificou-se que o tempo para atingir o máximo de conversão é maior em menores velocidades de agitação do meio reacional, devido ao processo de resistência à transferência de massa interferir na reação. Esse efeito foi eliminado a partir de $930 \mathrm{rpm}$, indicando que o processo passa a ser controlado pela reação química. Esse resultado é corroborado pelas imagens de MEV e pela estimativa da área superficial efetiva. A variação exponencial da razão $\mathrm{S}_{\mathrm{ef}} / \mathrm{S}_{\mathrm{o}}$ indica um aumento da reatividade da superfície das amostras de PETrSATT não reagidas devido ao aumento do número de ponto reativos na superfície. Esse aumento na reatividade da superfície diminui o tempo da reação para atingir altas conversões, principalmente a 170 e $185^{\circ} \mathrm{C}$. O modelo cinético adotado e a equação cinética deduzida tiveram uma boa concordância com os resultados experimentais. Os valores das constantes de velocidade aparente foram altas, comparadas com valores da literatura, indicando que o meio reacional alcalino adotado, o pré-tratamento dos "flakes" de PETr por imersão em água até a saturação e as condições de agitação utilizadas neste trabalho são vantajosos para serem usados como método de despolimerização do PETrSATT a TPA.

\section{Agradecimentos}

Ao CNPq (Processo 141263/2003-2) pela concessão da bolsa de doutorado para Priscila S. Curti.

Ao LIEC por disponibilizar o equipamento BET ASAP 2000, para as medidas de área superficial e ao técnico João Gonzalez pelas medidas.

Ao prof. Dr. Norberto Peporine Lopes, do Departamento de Física e Química da Faculdade de Ciências Farmacêuticas de Ribeirão Preto, Universidade de São Paulo - USP, pela análise de espectrometria de massas por electrospray. 


\section{Referências Bibliográficas}

1. www.cempre.org.br/fichas_técnicas_pet.php.

2. Awaja, F. \& Pavel, D. - Europ. Polym. J., 41, p. 1453 (2005).

3. Paszun, D. \& Spychaj, T. - Ind. Eng. Chem. Res., 36, p. 1373 (1997).

4. http://www.mgpolymers.com

5. Yoshioka, T.; Okayama, N. \& Okuwaki, A. - Ind. Eng. Chem. Res., 37, p. 336 (1998).

6. Yoshioka, T.; Motoki, T. \& Okuwaki, A. - Ind. Eng. Chem. Res., 40, p. 75 (2001).

7. Campanelli, J. R.; Kamal, M. R. \& Cooper, D. G. - J. Appl. Polym. Sci., 48, p. 443 (1993).

8. Campanelli, J. R.; Kamal, M. R. \& Cooper, D. G. - J. Appl. Polym. Sci., 54, p. 1731 (1994).

9. Kao C. Y., Wan B. Z. \& Cheng W. H. - Ind. Eng. Chem. Res., 37, p. 1228 (1998).

10. Wan B. Z. Kao C. Y. \& Cheng W. H. - Ind. Eng. Chem. Res., 40, p. 509 (2001).

11. Ruvolo-Filho A. \& Soares K., Patent, B. R., PI 04000749 (2004).
12. Oku, A.; Hu, L. C; \& Yamada, E. - J. Appl. Polym. Sci., 63, p. 595 (1997).

13. Goje, A. S. \& Mishra, S., - Macrom. Mat. Eng., 288, p. 326 (2003).

14. Mishra, S. \& Goje, A. S. - Polym. Reac. Eng., 11, p. 963 (2003).

15. Goje, A. S.; Thakur, S. A.; Diware, V. R.; Chauhan, Y. P. \& Mishra S. - Polym.-Plast. Techn. Eng., 43, p. 369 (2004).

16.Crotti, A. E. M.; Vessecchi, R.; Lopes, J. L. C.; Lopes, N. P. - Quím. Nova, 29, p. 287 (2006).

17. Morrison, R. T. \& Boyd, R. N. - "Organic Chemistry", Allyn and Bacon, 1973.

18. Sammon, C.; Yarwood, J. \& Everall, N. - Polym. Degrad. Stab., 67, p. 149 (2000).

19. Villain, F.; Coudane, J. \& Vert, M. - Polym. Degrad. Stab., 43, p.431 (1994).

20. Ruvolo-Filho, A. \& Carvalho de, G. M. - J. Macromol. Sci.-Phys., 38, p. 305 (1999).

21. Levenspiel, O. - "Engenharia das Reações Químicas Cálculo de reatores", Edgar Blucher, 1974, vol. 2.

22. Ruvolo-Filho, A. \& Curti, P. S. - Ind. Eng. Chem. Reser., submetido.

Enviado: 07/02/06

Reenviado: 05/07/06

Aceito: $24 / 07 / 06$ 